Submitted to the IEEE International Conference on Information, Intelligence and Systems (ICISS),

Washington, DC, Nov. 1-3, 1999

\section{Signal Trend Identification with Fuzzy Methods}

\author{
Xin Wang \\ School of Nuclear Engineering, Purdue University \\ W.Lafayette, Indiana47907-1290 \\ Email: wangx@purdue.edu \\ Thomas Y. C. Wei \\ Argonne National Laboratory, Reactor Engineering Division, Argonne, Illinois 60439 \\ Jaques Reifman \\ Argonne National Laboratory, Reactor Analysis Division, Argonne, Illinois 60439 \\ Lefteri H. Tsoukalas \\ School of Nuclear Engineering, Purdue University \\ W.Lafayette, Indiana47907-1290

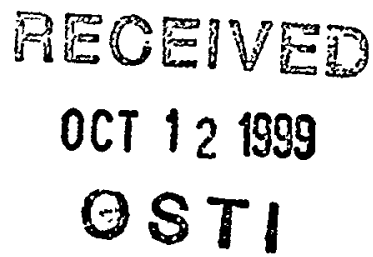 \\ U.S. Government purposes.
}

The submitted manuscript has been authored by a contractor of the U.S. Government under contract No. W-31-109ENG-38. Accordingly, the U.S. Government retains a nonexclusive, royalty-free license to publish or reproduce the published form of this contribution, or allow others to do so, for

\begin{abstract}
A fuzzy-logic-based methodology for on-line signal trend identification is introduced. Although signal trend identification is complicated by the presence of noise, fuzzy logic can help capture important features of on-line signals and classify incoming power plant signals into increasing, decreasing and steady-state trend categories. In order to verify the methodology, a code named PROTREN is developed and tested using plant data. The results indicate that the code is capable of detecting transients accurately, identifying trends reliably, and not misinterpreting a steady-state signal as a transient one.
\end{abstract}

\section{INTRODUCTION}

Signal trend identification is an important part of computer-based monitoring, diagnostic and control systems. In many applications it is an essential first step in the reliable and timely diagnosis of complex systems, such as nuclear power plants and industrial processes. Although conventional methods have been widely applied for signal trend identification, these methods are generally signal- and process-dependent, and hence, cannot be easily ported to other processes and plants. Here, we describe a new fuzzy-logicbased method, which is signal- and processindependent, that performs signal trend identification.

Argonne National Laboratory (ANL) and Purdue University are collaborating on the development of a novel operator advisory knowledge-based digital system called IGENPRO. ${ }^{1}$ It is an advanced plant- and thermal-hydraulic process- independent system for nuclear power plant transient diagnostics and management.

There are three major modules in IGENPRO: PROTREN, PRODIAG and PROMANA. The first module (PROTREN) performs signal processing. Each individual signal trend is classified as increasing, decreasing or constant and the results are fed to the second module. ${ }^{1,2}$ The second module (PRODIAG) performs plantlevel diagnostics. It is based on a knowledge base of generic thermodynamic first principles, such as mass, momentum, and energy conservation equations. The PRODIAG knowledge base does not follow a conventional event-based approach, but rather a generic function-based approach with a comprehensive although compact, knowledge base. ${ }^{3}$ The third module (PROMANA) recommends a series of operations for plant recovery.

In this paper, we describe the theoretical concepts of the fuzzy-logic-based PROTREN 


\section{DISCLAIMER}

This report was prepared as an account of work sponsored by an agency of the United States Government. Neither the United States Government nor any agency thereof, nor any of their employees, make any warranty, express or implied, or assumes any legal liability or responsibility for the accuracy, completeness, or usefulness of any information, apparatus, product, or process disclosed, or represents that its use would not infringe privately owned rights. Reference herein to any specific commercial product, process, or service by trade name, trademark, manufacturer, or otherwise does not necessarily constitute or imply its endorsement, recommendation, or favoring by the United States Government or any agency thereof. The views and opinions of authors expressed herein do not necessarily state or reflect those of the United States Government or any agency thereof. 


\section{DISCLAIMER}

Portions of this document may be illegible in electronic image products. Images are produced from the best available original document. 
Submitted to the IEEE International Conference on Information, Intelligence and Systems (ICIIS), Washington, DC, Nov. 1-3, 1999

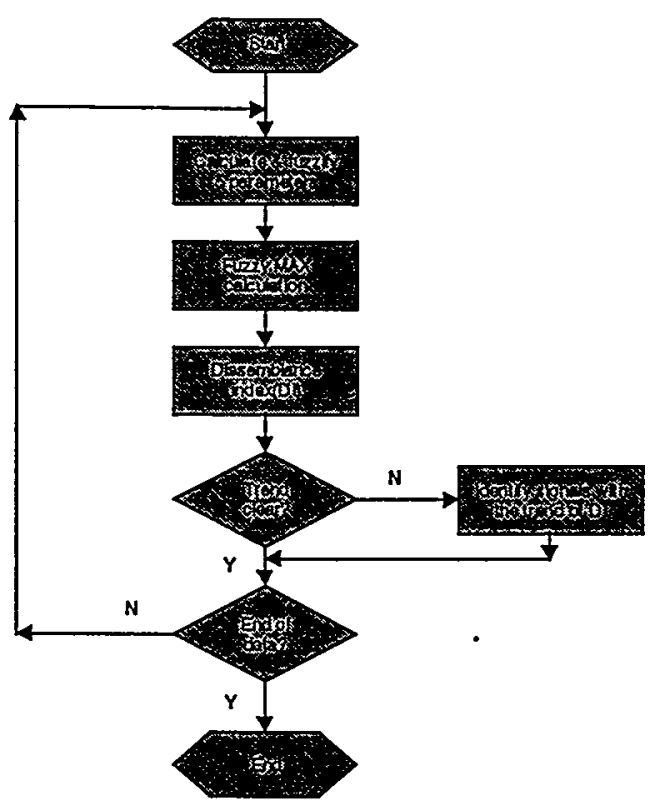

Fig.1. Flowchart of the methodology

\section{BACKGROUND AND BASIC CONCEPTS}

\section{Outline of the methodology}

For the purpose of on-line classification of an incoming plant signal trend as increasing, decreasing, or constant, some preprocessing needs to be performed to extract useful signal features. In PROTREN, five parameters representing several features of the signal are extracted. In order to incorporate all the information included in these features, the parameters are transformed into fuzzy numbers which are then synthesized into one final fuzzy number representing, in an approximate way, the degree to which the current constellation of features offers evidence for the onset of a transient. ${ }^{4}$ Then, based on the final fuzzy number and the use of fuzzy logic, a trend identification decision is made. The basic structure of the technology discussed below is shown in Fig.1.

\section{Development of final fuzzy number}

The information on signal trends is assumed to be represented by the final fuzzy number that summarizes the important features of the signal. This is performed through the following threestep process.

\subsection{Definition of the five parameters}

The definition of the five parameters is based on the assumption that during steady-state process operation the plant signals are normally distributed with mean value $\mu$ and standard deviation $\sigma$. The values of $\mu$ and $\sigma$ can be computed off-line based on steady-state historical data before they are used on-line, at 
each sampling time, to compute the five parameters. For each sampling signal, the mathematical description of the parameters is as follows:

\section{(a) Probability density function( $p d f$ )}

$$
p d f_{t_{c}}=\frac{1}{\sqrt{2 \pi} \sigma} \exp ^{-\frac{\left(s_{c}-\mu\right)^{2}}{2 \sigma^{2}}}
$$

where,

$t_{c}=$ current time step

$S_{t_{c}}=$ signal value at $t_{c}$

$\mu=$ mean value of the steady-state signal

$\sigma=$ standard deviation of the steady-state signal

Sample points belonging to off-normal states have small $p d f$ values. Thus, this parameter can determine the deviation of the signal from steady-state operation.

(b) Cumulative probability density function (cum_pdf)

$$
\text { cum }-p d f_{t_{c}}=\sum_{i=0}^{n} p d f_{t_{c-1}}
$$

where $n$ represents the length of the time window. Small signal changes are accumulated and recorded in this parameter. By accumulating small signal changes, it becomes possible to make decisions based on not only the instantaneous signal changes, e.g., the $p d f$, but also on the recent history of the signal.

\section{(c) Average derivative (avgd)}

$$
\operatorname{avgd}_{t_{c}}=\frac{\operatorname{avg} t_{c}-a v g_{t_{c-1}}}{t_{c}-t_{c-1}}
$$

where,

$$
\operatorname{avg}_{t_{c}}=\frac{\sum_{i=0}^{n}\left[e^{-k\left(t_{c}-t_{c-1}\right)} s_{t_{c-1}}\right]}{\sum_{i=0}^{n} e^{-k\left(t_{c}-t_{c-1}\right)}}-
$$

This parameter represents the time rate of change of the variable avg, which is defined as the weighted sum of the sampled signal values over the time window of length $n$. In Eq.(4), $k$ is a positive constant.

\section{(d) Relative deviation (ravg )}

$$
\operatorname{ravg}_{i_{e}}=\frac{a v g_{t_{c}}-\mu}{\mu}
$$

This parameter represents the deviation of avg from the signal mean steady-state value and is independent of the amplitude of the signal. The sign of this parameter is used to differentiate between increasing and decreasing trends.

\section{(e) Sample derivative ( $s d$ )}

$$
s d_{t_{c}}=\frac{s_{t_{c}}-s_{t_{c-1}}}{t_{c}-t_{c-1}}
$$

This parameter is used to capture the instantaneous rate of change of the original signal without any smoothing since smoothing hides the occurrence of local peaks.

\subsection{Fuzzification of the five parameters}

In order to synthesize the information described by the five parameters, they are transformed into fuzzy numbers and mapped into a $[0,1]$ truth domain. $^{4}$ 
Submitted to the IEEE International Conference on Information, Intelligence and Systems (ICIIS), Washington, DC, Nov. 1-3, 1999

As shown in the conversion table, Table.1, the truth of a parameter is computed according to its actual value. Then a fuzzy number is set up on the $[0,1]$ truth domain according to the conversion map.

Table.1. Conversion table for transforming a crisp number into a fuzzy number

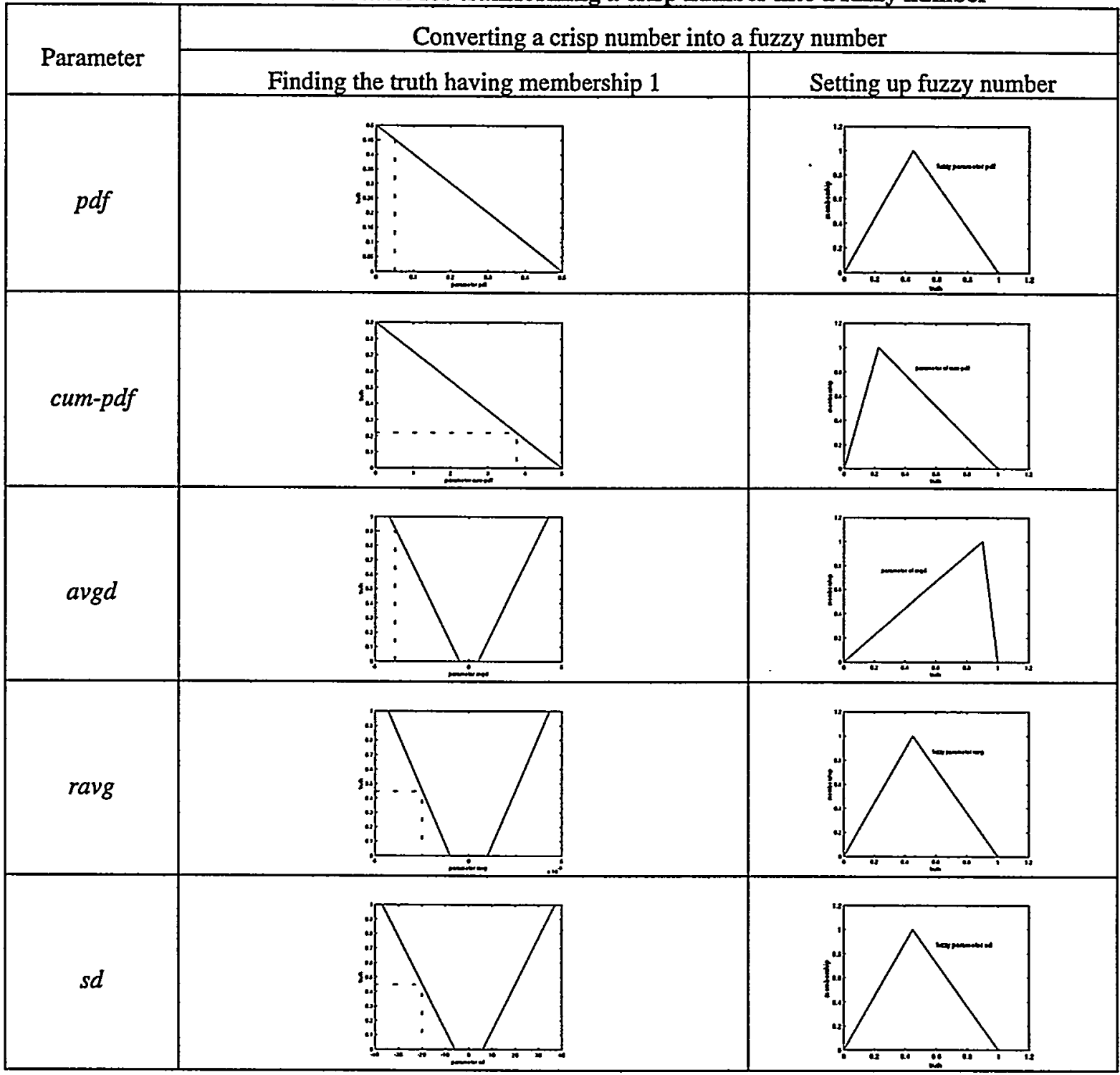


2.3. Formulation of the final fuzzy number with the MAX operation

A final fuzzy number is computed to incorporate the information provided by the five fuzzy numbers associated with the five parameters. Here, the final fuzzy number is computed by the MAX operator. ${ }^{5}$

$$
\left(a^{\alpha}, b^{\alpha}\right)=\left(\max _{i=1-5}\left(a_{i}^{\alpha}\right), \max _{i=1-5}\left(b_{i}^{\alpha}\right)\right)(7)
$$

where,

$a^{\alpha}=$ the left point of the of $\alpha$-cut of the final fuzzy number

$b^{\alpha}=$ the right point of the of $\alpha$-cut of the final fuzzy number

$a_{i}^{\alpha}=$ the left point of the of $\alpha$-cut of the $i$ th fuzzy number

$b_{i}^{\alpha}=$ the right point of the of $\alpha$-cut of the $i$ th fuzzy number

The final decision is made on the basis of this new fuzzy number.

\section{Trend evaluation}

The trend inference is obtained based whether the signal trend pertains to 'steady state' or 'transient state'. Because 'steady' and 'transient' are both fuzzy concepts, a fuzzy decision strategy is developed. The methodology has two parts: defuzzification and fuzzy decision.

\subsection{Computing the dissemblance index and the confidence confid}

In order to draw a conclusion concerning the final fuzzy number, i.e., defuzzification, the distance between the membership function of the final fuzzy number and prototype membership functions are calculated. The distance is also called the dissemblance index (DI) of two fuzzy numbers $A$ and $B, \delta(A, B)$, and is defined as: ${ }^{6}$

$$
\begin{aligned}
& \delta(A, B)=\int_{\alpha=0}^{1} \delta\left(A_{\alpha}, B_{\alpha}\right) d \alpha \\
& =\frac{1}{2} \beta \int_{\alpha=0}^{1} \Delta\left(A_{\alpha}, B_{\alpha}\right) d \alpha \\
& =\frac{1}{2} \beta \int_{\alpha=0}^{1}\left(a_{1}^{\alpha}-b_{1}^{\alpha}|+| a_{2}^{\alpha}-b_{2}^{\alpha} \mid\right) d \alpha
\end{aligned}
$$

where,

$\beta$ is used to normalize the value of DI to $[0,1]$

$a_{1}^{\alpha}\left(b_{1}^{\alpha}\right)=$ the left point of the $\alpha$-cut of a fuzzy number of $A(B)$

$a_{2}^{\alpha}\left(b_{2}^{\alpha}\right)=$ the right point of the $\alpha$-cut of a fuzzy number $A(B)$

The two prototype membership functions are fuzzy numbers 0 and 1 which represent steady state and transient signals respectively. The Zadeh diagrams of these numbers are shown in Fig. ${ }^{4}$ 


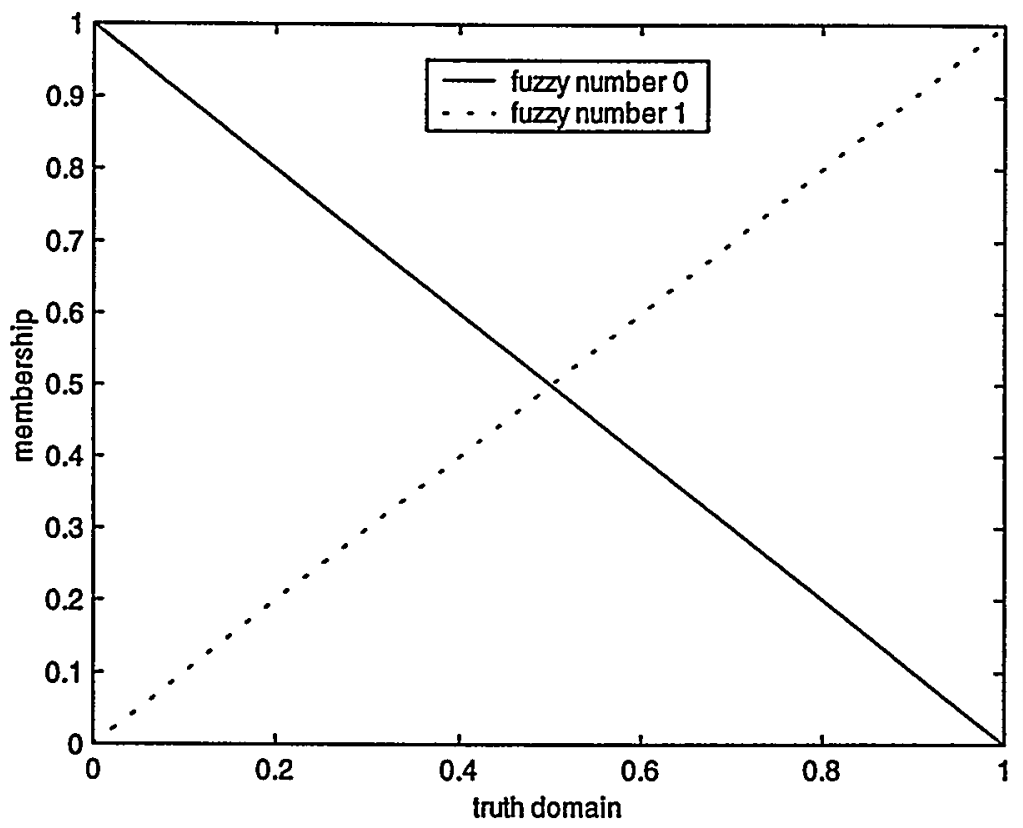

Fig.2. Fuzzy number 0 and 1

Next, we define the parameter confid, which is used to infer the confidence in the decision.

$$
\text { confid }=\frac{\delta_{1}}{\delta_{0}+\delta_{1}}
$$

where,

$\delta_{l}=$ distance or DIfrom the final fuzzy number to fuzzy 1

$\delta_{0}=$ distance or DI from the final fuzzy number to fuzzy 0

Two decision methods in which the parameter confid plays different roles are considered. The first one is a non-fuzzy decision method while the second one is a fuzzy decision method.

In the non-fuzzy decision method, the decision is made on the basis of the value of confid. If the value of confid is larger than 0.5 , it means that the final fuzzy number is closer to 0 than to 1 indicating a steady-state trend. On the other hand, a confid of less than 0.5 indicates a changing signal trend.

Because of the crisp nature of this decision method, under certain conditions, it can provide unstable (i.e., oscillatory) trend inferences. For example, if the signal trend is not well established, the value of confid can oscillate about 0.5 , resulting in conflicting inferences. To avoid this undesirable behavior, we propose the use of the following fuzzy decision strategy.

\subsection{Fuzzy decision strategy}

The fuzzy decision strategy is composed of several rules. First, we use three rules to identify the trend of the signal:

- If confid indicates that the fuzzy number is apparently close to fuzzy number 0 , then the signal trend is not changing.

- If confid indicates that the fuzzy number is apparently close to fuzzy number 1 , then the signal trend is changing.

- If the decision cannot be made confidently, i.e., $\delta_{0}$ is close to $\delta_{1}$, then the history of the change in confid is used.

According to these rules, a decision is made only when confid is close to 0 or 1 , i.e., the result is relatively clear. If the final fuzzy number falls 
within the fuzzy region as mentioned in the third rule, two additional rules are used.

- If the value of confid is continuously decreasing, then the signal is not constant.

- If the value of confid is oscillating or increasing, the signal is assumed to be constant.

One major characteristic of the strategy is that the decisions made are not only dependent on the values of $\delta_{0}, \delta_{1}$ and confid, but also on the previous values of confid. Actually, in many cases, the changing trend of confid is even more important than the parameter itself.

For example, suppose $\delta_{0}$ is a little less than $\delta_{l}$, but the value of confid is decreasing continuously throughout the last sample points. In this case, $\delta_{0}$ larger than $\delta_{l}$ can be predicted to occur within the next few steps and a changing inference can be made. On the other hand, if the value of confid is oscillating violently, the strategy most likely identifies a signal as steady state even when $\delta_{0}$ larger than $\delta_{l}$.

Monitoring the past values of confid does not impair the performance of the overall strategy. Off-line signal analysis shows that the changing trend of confid for steady-state is much different than that of transient state even for slow and small changes. Therefore, this strategy is very useful in determining to which state the current sample point pertains and contributes to quicker response and more stable results.

\section{RESULTS}

This methodology has been incorporated into the code PROTREN, which is the signal processing module of the computer-based diagnostics and management system IGENPRO.

Figures 3 and 4 show two flow signal data from the Chemical Volume Control System of a pressurized water reactor power plant sampled at 5 s intervals. Fig. 3 illustrates the changing pump discharge header flow and Fig.4 illustrates the outlet flow of the letdown heat exchanger.

The signal shown in Fig. 3 is a transient one. PROTREN begins to provide the transient result about 15s after the onset, when the change is about $0.3 \%$. It is apparent that the methodology does not deduce transients for steady state signals and can provide detection of transients for actual transient signals.

Due to the difficulties involved in obtaining actual data from power plants, many validation experiments with simulated signals were performed. Although it is difficult for conventional methods to differentiate between small and slowly changing transient signals and noisy steady-state signals, PROTREN can make correct and stable decisions. Figures 5 shows the PROTREN response to simulated data representing a slowly increasing signal trend. It takes PROTREN about ten minutes to get the correct and stable decision. Figure 6 shows the response for a fast transient resulting in a small magnitude increase in the signal value. In this case the correct inference is made in one time step from the onset of the transient and without any oscillatory behavior. 
Submitted to the IEEE International Conference on Information, Intelligence and Systems (ICIS), Washington, DC, Nov. 1-3, 1999
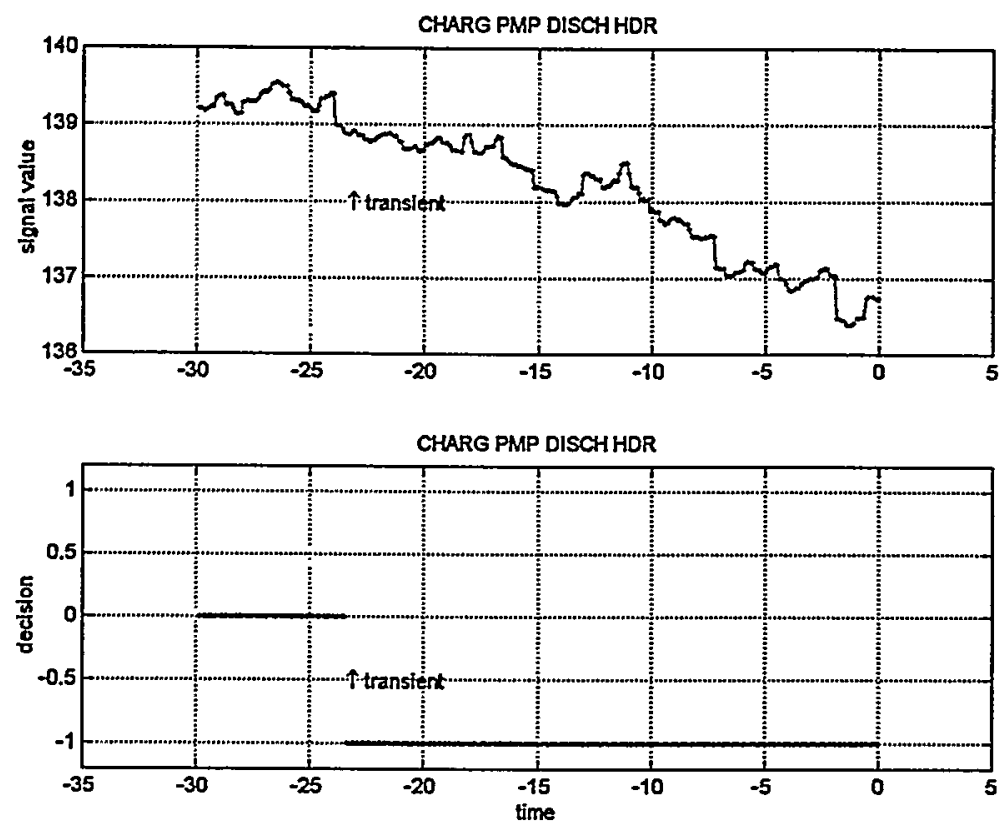

Fig.3. PROTREN results for a decreasing signal trend
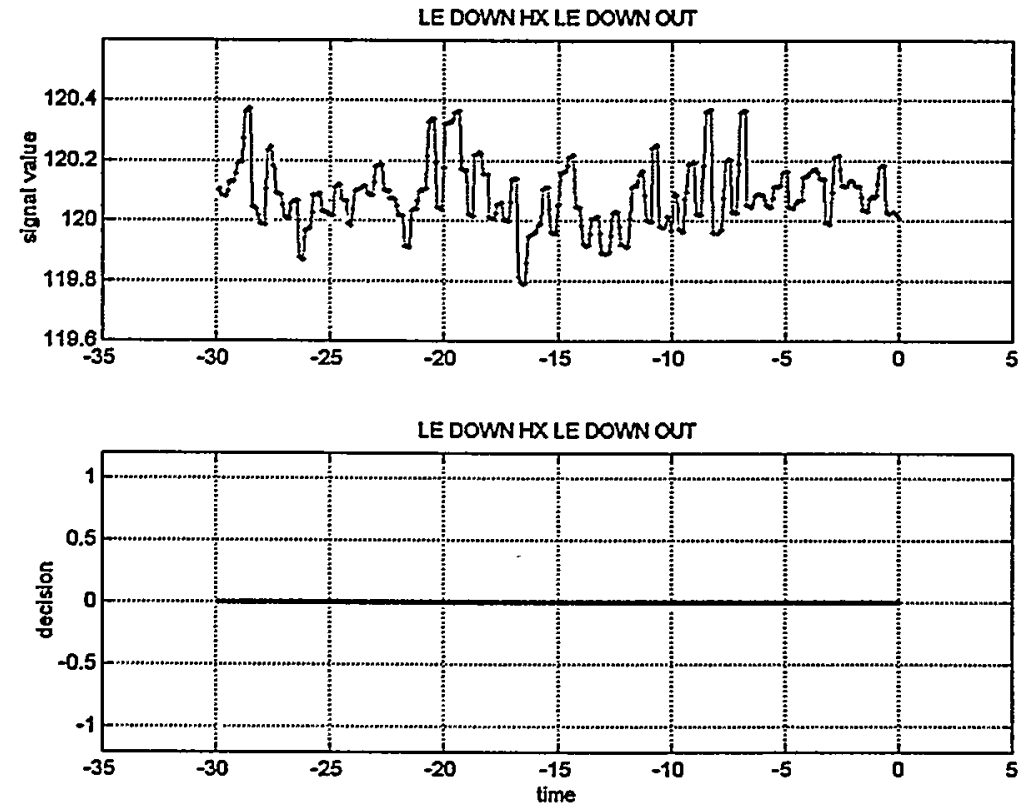

Fig.4. PROTREN results for a unchanging signal trend 
Submitted to the IEEE International Conference on Information, Intelligence and Systems (ICIIS), Washington, DC, Nov. 1-3, 1999
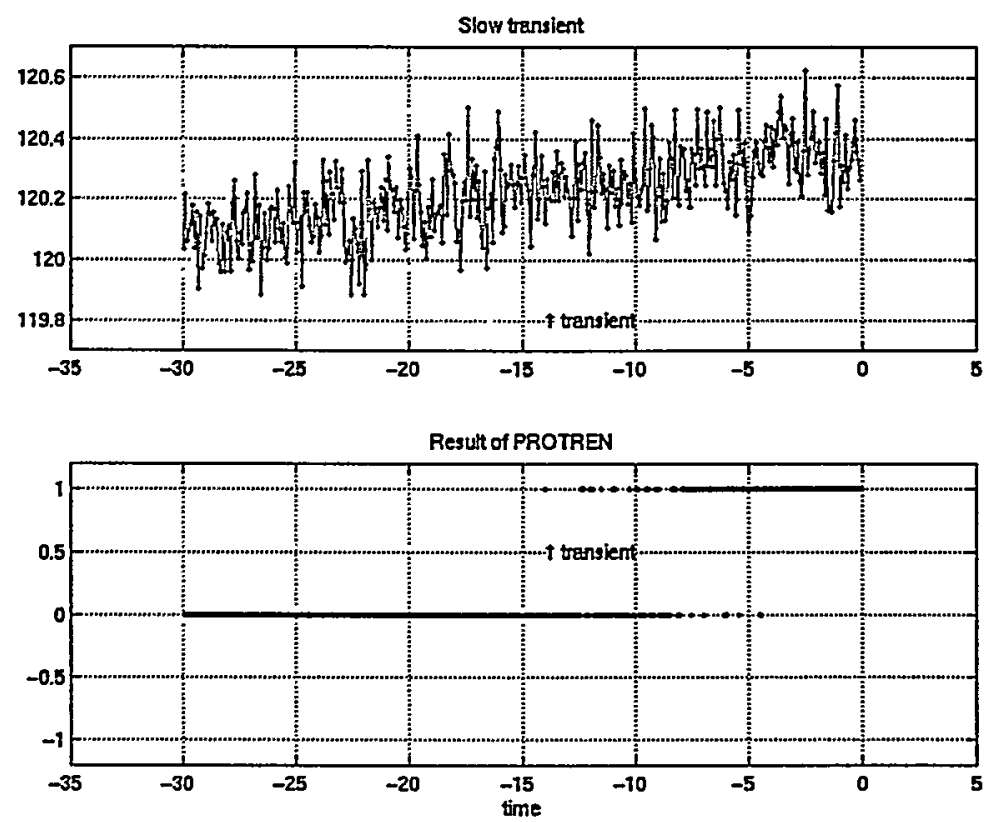

Fig.5. PROTREN results for a slowly increasing signal trend
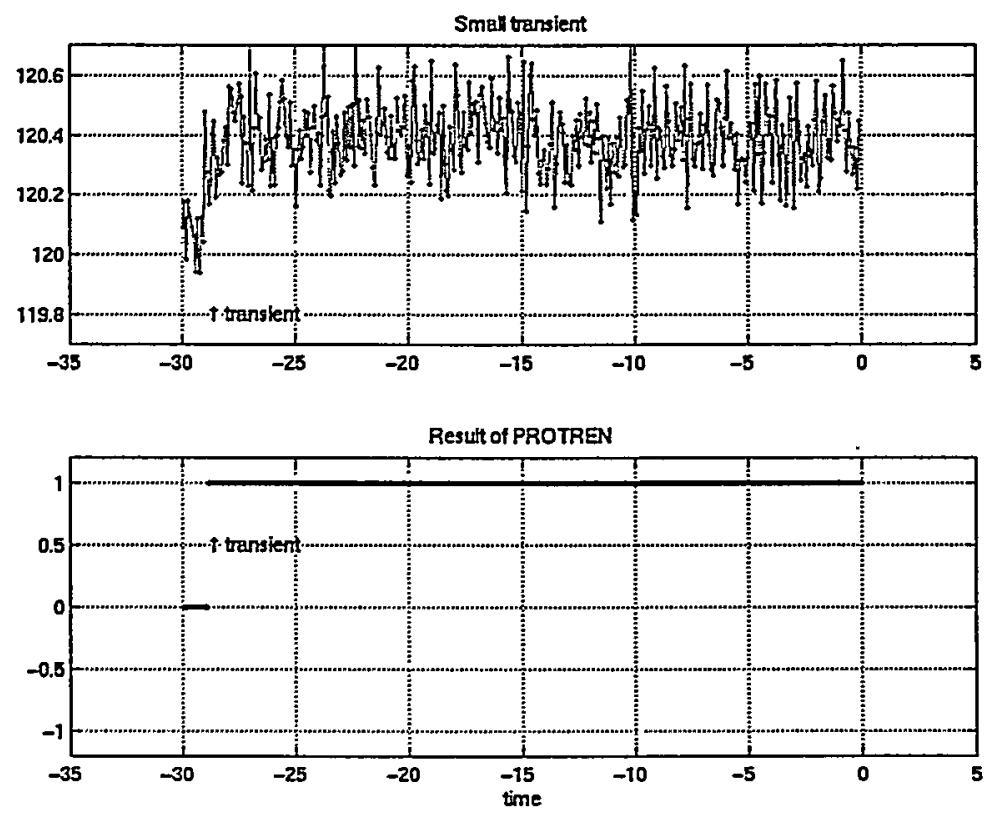

Fig.6. PROTREN results for a fast but small signal trend 


\section{CONCLUSIONS}

A fuzzy-logic-based methodology for signal trend identification is presented in this paper. It differs from conventional methods in several aspects.

- In order to perform on-line estimation of signal trends, five parameters are defined and transformed into a final fuzzy number. The trend identification is based on the analysis of the final fuzzy number.

- A fuzzy decision strategy is used to infer the signal trend when the final fuzzy number falls within a fuzzy region.

- Signals with changing trends can be deduced quickly and instabilities in the final decision are reduced significantly. At the same time, signals with unchanging trends are not misinterpreted.

PROTREN has been validated through numerous tests including both simulated and actual plant data. Preliminary results indicate that the proposed method is capable of early identification of signal trends in the presence of noisy data. Future research will involve further improvement of the algorithm and qualitative determination of its sensitivity.

\section{ACKNOWLEDGMENTS}

Work is supported by the U.S. Department of Energy, Office of Nuclear Energy, Science and Technology, under contract W-31-109-ENG-38. The authors also wish to thank Commonwealth Edison Company for providing plant signal data.

\section{REFERENCES}

1. J. A. Mormam, J. Reifman, J. E. Vitela, T. Y. C. Wei, C. A. Applequist, P. Hippley, W. Kuk and L. H. Tsoukalas, "IGENPRO Knowledge-Based Digital System for Process Transient Diagnostics and Management," Proceedings of the IAEA Meeting on Advanced Technologies for Improving Availability and Reliability of Current and Future Water Cooled Nuclear
Power Plants, Argonne, IL, September 811, 213-224, 1997.

2. J. Reifman, "Survey of Artificial Intelligence Methods for Detection and Identification of Component Faults in Nuclear Power Plants," Nuclear Technology, 119, 76-97, 1997.

3. Jaques Reifman, Thomas Y. C. Wei, "PRODIAG: A Process-independent Transient Diagnostic System-I: Theoretical Concepts," Nuclear Science and Engineering, 131, 1-19, 1999.

4. Jin Chai and L. H. Tsoukalas, "An Investigation of Fuzzy Trend Algorithms for Nuclear Power Plant Transients," School of Nuclear Engineering, Final Report, 1998.

5. Earl Cox, The Fuzzy Systems Handbook, Boston, 1994.

6. Arnold Kaufmann and Madan M. Gupta, Introduction to Fuzzy Arithmetic: Theory and Application, New York, 1991. 